\title{
Theoretical, physical and metrological problems of further development of measurement techniques and instrumentation in science and technology
}

\author{
D. Hofmann, Y.V. Tarbeyev \\ Friedrich -Schiller- University, 32 Ernst-Thälmannring, 69 Jena, GDR
}

This is a reissue of a paper which appeared in ACTA IMEKO 1979, Proceedings of the $8^{\text {th }}$ IMEKO Congress of the International Measurement Confederation, "Measurement for progress in science and technology", 21-27.5.1979, Moskow, vol. 3, pp. 607-626. Common interest of both metrologists and representatives of science and technology in constant improvement of measurements as well as general trends in the development of research in the field of metrology, measurement technology and instrumentation at the present-day stage are shown. Problems of general metrology, of improving systems of units and standards ("natural" standards in particular) are considered in detail.

\section{Section: RESEARCH PAPER}

Keywords: metrology, measurement technology, measurement theory, standards, fundamental physical constants, uniformity of measurements

Citation: D. Hofmann, Y.V. Tarbeyev, Theoretical, physical and metrological problems of further development of measurement techniques and instrumentation in science and technology, Acta IMEKO, vol. 3, no. 1, article 7, May 2014, identifier: IMEKO-ACTA-03 (2014)-01-07

Editor: Luca Mari, Università Carlo Cattaneo

Received May $1^{\text {st }}$, 2014; In final form May $1^{\text {st }}$, 2014; Published May 2014

Copyright: @ 2014 IMEKO. This is an open-access article distributed under the terms of the Creative Commons Attribution 3.0 License, which permits unrestricted use, distribution, and reproduction in any medium, provided the original author and source are credited

\section{INTRODUCTION}

The conversion of science into a major productive force is inevitably accompanied with growing interest in increasing measurement accuracy. Many objective studies of reality are reduced to measurements. At the same time the results of scientific research serve as a basis for the further development of this reality.

Experimenters in science and practical engineers are interested in higher measurement accuracy, in technical improvement of measuring instrumentation and in higher reliability of measurements. These aspects make up the object of metrology.

The task of meeting the urgent requirements of science and technology is the motive force for the progress of metrology and improvement of systems of units and standards which in the long run should provide for an optimum metrological assurance. This capacious concept includes not only the establishment of standards per se but also the development of most efficient methods of dissemination of the values of units and metrological supervision of the correctness of measurements as well as the development of up-to-date measuring transducers, fast automatic measuring systems that require minimum metrological supervision [1].
Metrology is a science, whose scope of knowledge is extremely polytechnical.

It should embrace a wide range of electromagnetic, optical, mechanical, physical and chemical, nuclear and many other phenomena and incorporate an immense number of measuring problems that cover measurement transformations, estimation of measurement results and uncertainties, realization of the units of physical quantities and their dissemination. Therefore, it is not difficult to foresee that the task of establishing metrology as an independent science enjoying full rights and having its own large domain of specially arranged knowledge, theorems, methods of investigation, is complicated.

The practical requirements and continuous growth of the role of measurements urgently demand that the progress of metrology must be accelerated. The aim of the present paper is to show the directions and ways of the further development of metrology. As examples you will become acquainted with some actual problems of modern metrology and measurement theory.

\section{COMMON TRENDS IN THE DEVELOPMENT OF RESEARCH AND APPLICATION IN THE FIELD OF METROLOGY AND MEASUREMENT TECHNOLOGY}

In the 20th century natural, technical and social sciences have become a decisive productive force. As a result of such 
sharp increase in the practical role of sciences, more and more technical subjects go over from empirically descriptive representation of the observed facts to generalizing theoretical considerations and concepts. In doing so, the peculiar features of the modern progress in scientific research are:

- a sharp increase in the use of systems approach to all problems

- an increase in the scope and intensity of fundamental research in the total volume of research work.

The above-mentioned statements are not only valid for metrology, but are also especially characteristic of the present stage in its development. It is determined by substantial qualitative and quantitative changes.

The most prominent quantitative changes are:

1. An increase in the number of measurements and in the number of fields in which measurements are employed. Practically, there are no technical fields where measurements are not made; the measurement expenses reach 50 to $60 \%$ of the total production expenses in the most advanced industries (Figure 1) [2].

2. Measurements provide indispensable technical key information for evaluating and controlling specialized cooperative production. They are carried out on a large scale and provide the decision criteria for acceptance or refusal of labor having been realized in masses.

3. Natural, technical and social sciences operate an instrument technique possessing industrial dimensions and demanding universal knowledge as well as a concerning behaviour of the scientists (in the fields of nucleonics, microelectronics, space research, radiology, electron-scan microscopy, psychological behavior research). Planned experiments are intended to prove the correctness of theoretical considerations.

4. Measurements have two main functions:

- an increased observability of preferred technical-physicalchemical states or procedures, widely exceeding the natural limit being set to the sensing organs of the measuring persons.
- the objectivation of observations made by measuring persons. It is achieved by comparing measured quantities with known and settled standards.

Qualitatively new requirements concerning measurement theory are additionally caused by the following facts:

5. The accuracy of measurements required both in scientific experiments and in industry is more and more approaching the level of standard measurements.

6. Due to the high sensitivity of measuring instruments the environmental conditions and external influencing factors are producing ever-increasing effects on the measurement results.

7. Sophisticated measuring systems whose calibration by traditional methods presents big problems are becoming widespread.

8. Greater sophistication of measuring instrumentation in respect of both the operating principle and the design features makes still higher demands of its operators.

Thus, today in measurements, measurement technology and instrumentation take place those qualitative and quantitative changes which not only require the proper investigation of individual metrological problems but also inevitably result in an urgent need for the development of a general theory of metrology and, first of all, a general (unified) theory of measurements.

The development of a general measurement theory is complicated by the following state of affairs:

1. Measurement science has a long history and therefore a mighty tradition. In our days this tradition is still aimed at a further differentiation.

2. Measuring has two different definitions:

- measuring in a narrow sense, that is experimental comparing of a measured quantity to a known comparison quantity of the same kind (quality) which had been determined to be the unit of measurement. The measurement of physical quantities and the use of ratio scales are typical procedures. (Figure 2) [3].

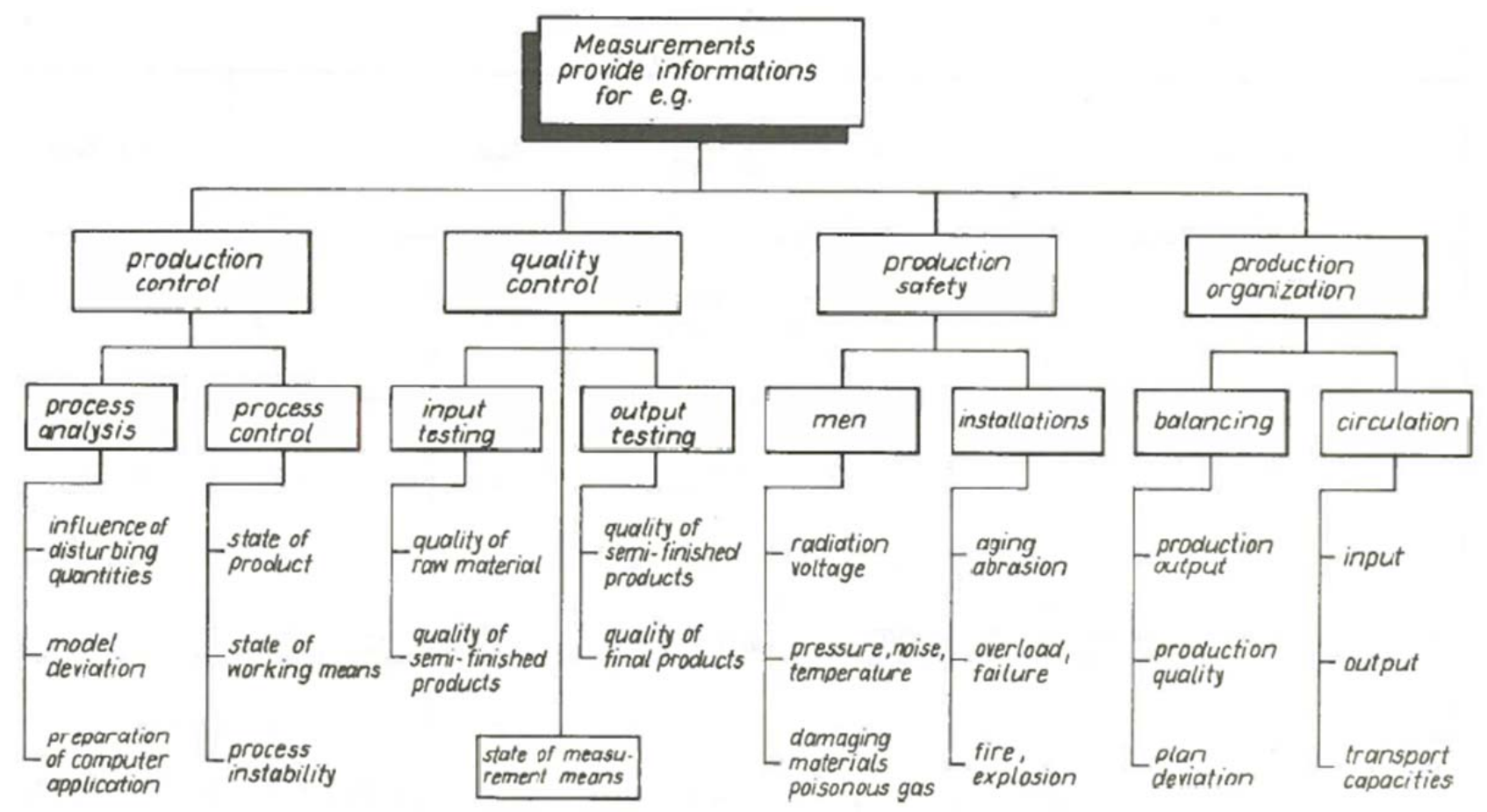

Figure 1. Acquisition of measurement information in production. 
- measuring in a wide sense is the coordination of numbers with states or procedures, following some rule. Scaling technical and no technical matters without special knowledge and using low value scales are typical procedures (Figure 2) [3].

At measuring in a narrow sense the subjective influence of the measuring person can little by little be diminished. This does not apply to measuring in a wide sense. At measuring you will observe disadvantages and inaccuracies if it is not considered

- that each quantity is always a quantity of a certain quality

- that the coordination of numbers with objects is also possible without knowing their quality

- that formal operating with numbers for any data you like (measured values or non-measured values) is possible and

- that mathematical models cannot express themselves if they represent correct facts or arbitrary phenomena [3, p. 37].

3. Historically we are just beginning to provide generalized knowledge in the fields of measurement engineering and measurement theory. The example of mass measurement is to prove that.

Already 2500 B.C. the equal-armed balance had been graphically represented in a pyramid in Gizeh in ancient Egypt. About 300 B.C. Aristotle, Euclid and Archimedes provided the theory for this balance. Electromechanical balances, with strain gages and radiometric balances are developments of the 20th century [4,5].

4. The special language of measurement engineering comprises a great number of terms. Numerous terms have not yet been well defined. Synonyms and polysems are frequently used [5]. Classifications and teaching conceptions in measurement engineering have the following points of view:

- measured quantities (length, force, temperature measurement engineering)

- measuring devices (strain-gage, oscillograph and digital measurement engineering)

- measurement principles (isotope, ultrasonic, infrared measurement engineering)

- field of measurement (production, process, laboratory measurement engineering).

\section{PROBLEMS OF THE GENERALIZED MEASUREMENT THEORY}

Topical tasks of measurement theory are

1. To increase the uniformity of measurements

2. To assure correct measurements

3. To get valid models of measurements and its aims.

Working with models has methodical advantages. In contrast to the originals (measured signals, measuring systems and measurement processes) models are simpler, cheaper, more easily described, can be transformed in time and space, varied, limited and optimized.

As a rule, modelling is associated with a certain simplification of the original. For example, a certain body is characterized by the following properties: length, surface roughness, volume, mass, density, temperature, colour, etc. Usually, only selected parameters of the object are considered exactly. Physical (homolog) models transform the scale of the original only or just simplify the original; the measured quantities of the original and model coincide. Mathematical (analog) models describe similar (analog) processes in different fields of knowledge; the measured quantities of the original and model do not coincide.

Models consist of a certain number of elements and relations (links) between them. A great advantage is that in gaining knowledge and building up a theory the originals of different nature can produce similar images that allow a uniform treatment and interpretation.

In any case the knowledge that was gained using a model in the image field should be confirmed in practice for the actual object. Otherwise the value of this knowledge is subject to argument.

The measurement theory deals mainly with well-defined or poorly-defined models of behaviour [6] expressed as algorithms. These models describe:

- measured signals and measuring systems in terms of mathematical algorithms,

- measurement processes in terms of heuristic algorithms.

\begin{tabular}{|c|c|c|c|c|c|c|}
\hline scale & $\begin{array}{l}\text { prefered fields } \\
\text { of application }\end{array}$ & $\begin{array}{l}\text { contents of } \\
\text { information }\end{array}$ & suitable for & $\begin{array}{l}\text { empirical } \\
\text { operations }\end{array}$ & $\begin{array}{l}\text { permitted } \\
\text { transformations }\end{array}$ & $\begin{array}{l}\text { testing } \\
\text { characteristics }\end{array}$ \\
\hline nominal & social sciences & small & $\begin{array}{l}\text { qualitative } \\
\text { classification }\end{array}$ & $a=b ; a+b$ & $a b ; b a$ & attribute \\
\hline ordinal & $\begin{array}{l}\text { economic } \\
\text { sciences }\end{array}$ & medium & $\begin{array}{l}\text { quantitative } \\
\text { classification }\end{array}$ & $a<b<c<a$ & $y=f(a, b, c, d)$ & attribute \\
\hline interval & $\begin{array}{l}\text { technical } \\
\text { sciences }\end{array}$ & large & $\begin{array}{l}\text { relative } \\
\text { measurement }\end{array}$ & $\overline{a b} \cdot \overline{b c} \cdot \overline{c d}$ & $y=k_{1}+k_{2} \overline{a b}$ & variable \\
\hline ratio & $\begin{array}{l}\text { natural } \\
\text { sciences }\end{array}$ & very large & $\begin{array}{l}\text { absolute } \\
\text { measurement }\end{array}$ & $\frac{b}{a}-\frac{c}{b}-\frac{d}{c}$ & $y=k \overline{a b}$ & variable \\
\hline
\end{tabular}

Figure 2. Classification of scales and their properties after [3]. 
Specific features of mathematical algorithms are determinism, finiteness, universality and guarantee of solution [7].

Heuristic algorithms are not associated with such strict requirements and still they guarantee only a solution probability $0<\mathrm{p}(\mathrm{L})<1$ which tends to $1[8]$.

Both mathematical and heuristic algorithms take the shape of measurement rules or definitions. One should bear in mind that mathematical relationships obtained by a deductive reasoning cannot be used for scientific substantiation of metrological characteristics if they do not agree with the empirical knowledge (Figure 3) [9-11].

There are no good or bad models. A model can be considered a good one if it meets the practical requirements and is in good agreement with empirical data.

There is always the danger of:

- using too simple or too complicated models;

- solving partial problems only for which certain models are known;

- ascribing greater significance to those stages of processes that are described by mathematical algorithms than to those that can for the time being be described in terms of heuristic algorithms only.

Here are some typical examples of this sort:

- in the preference of random errors rather than systematic ones in the measurement error analysis;

- in the description of a measurement process the preference of an estimate to the detriment of the measurement process itself, with the measurement preparation being almost totally ignored;

- the preference of the normal distribution even when considering events whose distribution differs from the normal one;

- the preference of incompletely formulated measurement problems (research problems) rather than completely formulated measurement problems (repetition problems);

- the neglecting of ergonomic aspects when developing new measuring instruments or processes;

- the neglecting of economic consequences as a result of incorrect measurements or the absence of measurements.

Operations (actions, decisions) aimed at an optimum solution of measurement problems are called the measurement strategy.

As a rule, the measurement strategy consists of numerous mathematical and heuristic algorithms. An algorithm transforms the input quantities into output quantities in accordance with a system of rules. The use of algorithms is inevitable for automation of measurement processes. The measurement strategy should cover the preparation, realization and evaluation of measurements (Figure 4) [2].

When one looks up in metrological literature for possible ways of solving measurement problems, one discovers a totally unsatisfactory situation. First of all, there is no clarity as to the necessary contents of measurement problems with guaranteed solution. The same applies to the choice of measuring instrumentation and the construction of measuring systems [12].

Mathematization of the measurement theory is, first of all, of great importance for microprocessor application. Neugebauer is of the opinion [13, p.44] that mathematics originated from metrological problems. In any case, afterwards it developed by a deductive method independently of metrological and other technical problems and at present it has in part surpassed the limits of practical problems.

A sharply increasing interest in mathematization of measurement processes results in a greater interest for publications on the axiomatic foundation of measurements. We are speaking here about literature devoted to the algorithmic

\begin{tabular}{|c|c|c|c|c|}
\hline Range Naming & $\begin{array}{l}\text { Input } \\
\text { quantity }\end{array}$ & Characteristics, Characteristic Function. & $\begin{array}{l}\text { Output } \\
\text { quantity }\end{array}$ & $\begin{array}{l}\text { Derived } \\
\text { quantity }\end{array}$ \\
\hline \multirow{4}{*}{$\begin{array}{l}\text { Time range } \\
\quad t, \tau\end{array}$} & $x_{e}(t)$ & 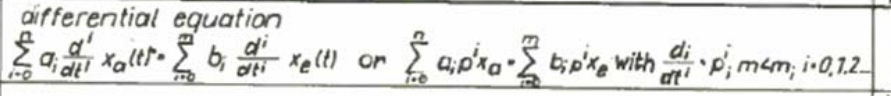 & $x_{a}(t)$ & \\
\hline & $\begin{array}{l}\text { Unit step } \\
\text { function } \\
1(t)\end{array}$ & & $\begin{array}{l}\text { transient } \\
\text { function } \\
h(t) \cdot \int g(t) d t\end{array}$ & \multirow{2}{*}{$\begin{array}{l}\text { response time } \\
t_{E} \cdot \frac{1}{2 f g} \\
\text { channel capacity } \\
C_{T} \cdot \frac{3}{t_{E}}\end{array}$} \\
\hline & $\begin{array}{l}\text { Unit collision } \\
\text { function } \\
\delta(t)\end{array}$ & & $\begin{array}{l}\text { weight function } \\
g(t) \cdot \frac{d h(t)}{d t}\end{array}$ & \\
\hline & 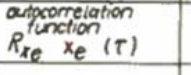 & 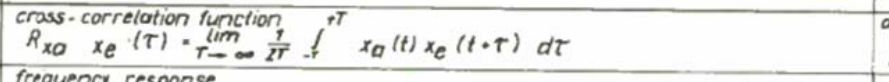 & $\begin{array}{l}\text { antocorreflation } \\
R_{x a \times a}^{\text {function }}(\tau)\end{array}$ & \\
\hline \multirow{4}{*}{$\begin{array}{l}\text { Frequency } \\
\text { range } \\
\qquad \omega\end{array}$} & \multirow{4}{*}{$\tilde{x}_{e}(j \omega)$} & $\begin{array}{l}\text { frequency response } \\
S(j \omega)=\frac{\tilde{x}_{a}(j \omega)}{\tilde{x}_{e}(j \omega)} \cdot \frac{F\left(x_{a}(t)\right]}{f\left(x_{e}(t)\right\}} \cdot \frac{f\left\{R_{x_{e}} x_{e}(t)\right]}{f\left\{R_{x_{e}} x_{e}(r)\right\}} \cdot S(\omega) e^{j p(\omega)}=\frac{\hat{x}_{a}}{\hat{x}_{e}} \cos \varphi \cdot j \frac{\hat{x}_{a}}{\hat{x}_{e}} \sin \varphi=A(\omega)+j B(\omega)\end{array}$ & \multirow{4}{*}{$\tilde{x}_{a}(j \omega)$} & \multirow[b]{2}{*}{$\begin{array}{l}\text { limiting rrequency } \\
f g \cdot \frac{1}{2 t_{\varepsilon}}\end{array}$} \\
\hline & & $\begin{array}{l}\text { amplitude response } \\
S(\omega)=|S(j \omega)|=\sqrt{A^{2}(\omega)+B^{2}(\omega)}=\frac{\hat{\lambda}_{C}(\omega)}{\hat{\lambda}_{C}(\omega)} \text { für } \quad 0 \leq \omega<\infty\end{array}$ & & \\
\hline & & $\begin{array}{l}\text { phase resporse } \\
\varphi(\omega)=\arg S(j \omega)-\arctan [E(\omega) / A(\omega)]-\varangle \hat{x}_{O}(\omega), \hat{x}_{e}(\omega) \text { für } 0 \leqslant \omega<\infty \\
\text { power - density spectrum }\end{array}$ & & \\
\hline & & $\begin{array}{l}\text { power - density spectrum } \\
P(\omega) \cdot\left|S(j \omega)^{2}\right|-S(j \omega) S(-j \omega)\end{array}$ & & \\
\hline $\begin{array}{l}\text { Complex } \\
\text { Frequency range } \\
\qquad p\end{array}$ & $\tilde{x}_{e}(\rho)$ & $S(p)=\frac{\tilde{x}_{a}(p)}{\tilde{x}_{e}(p)} \frac{\sum b_{i} p^{i}}{\sum a_{i} p^{i}} \cdot \frac{L\left\{x_{a}(t)\right\}}{L\left[x_{e}(t)\right\}} \cdot L\{g(t)\} \cdot \rho L\{h(t)\}$ & $\tilde{x}_{a}(p)$ & \\
\hline Designation & \multicolumn{2}{|c|}{ 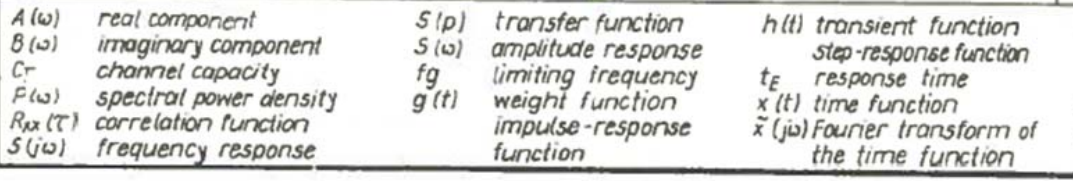 } & \multicolumn{2}{|c|}{$\begin{array}{l}\tilde{x}(0) \text { Laplace transform of the } \\
\text { time function } \\
1(t) \text { unit step function } \\
\delta(t) \text { Dirac function } \\
\varphi(\omega) \text { phase response }\end{array}$} \\
\hline
\end{tabular}

Figure 3. Characteristic functions of dynamics properties of measurement systems. 


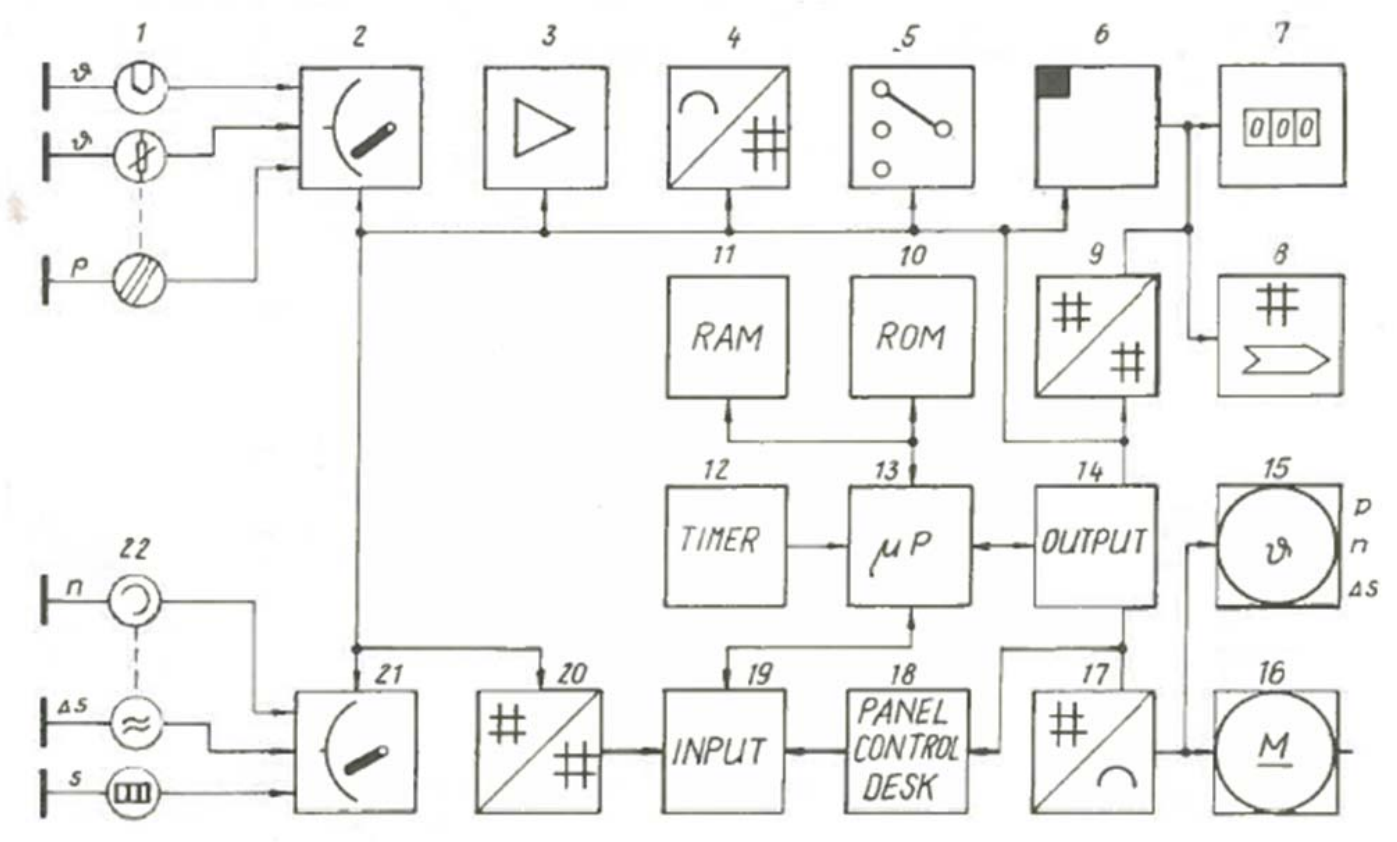

\begin{tabular}{lllll}
\hline 1 analog sensor & 6 memory & 11 random access memory & 16 final control element 21 scanner \\
2 & scaminer & 7 digital display & 12 timing generator & 17 D converter \\
3 amplifier & 8 printer, puncher & 13 microprocessor & 18 control desk \\
4 AlD converter & 9 code converter & 14 display unit & 19 input unit \\
5 signalscanner & 10 read only memory & 15 screen display device & 20 code converter
\end{tabular}

Figure 4. Components and functions of automatic measuring devices.

theory of measures and measurement theory [14-17].

The algorithmic theory of measures deals with the determination of the contents of geometrical structures or point sets. It is especially important for integral or probability calculations. In integral calculations the use of a measure instead of the contents results in the Lebesgue integral (expansion of the Riemann integral).

When estimating the probability, one random event is interpreted as a particular quantity $\mathrm{x}_{\mathrm{ai}}$ of the set of all elementary events and the measure $\mathrm{p}\left(\mathrm{x}_{\mathrm{ai}}\right)$ is considered to be the probability of the event $\mathrm{x}_{\mathrm{ai}}$.

In mathematics the point sets having the contents are called measurable. The prerequisite is the possibility of generating a set $\mathrm{M}$ of natural numbers and their resolution. This is true, for example, for the set of all even numbers, Fibonacci numbers and prime numbers which can be produced recursively.

The algorithmic theory of measurements proceeds from the fact that non-numerical algorithms also can be reduced to recursive functions and recursive sets of natural numbers if the class $\mathrm{K}$ of non-numerical input and output quantities can be coded using natural numbers.

For this purpose it is necessary to have:

- an algorithm that controls the coding;

- an algorithm that checks whether a certain natural number is the image of a non-numerical object from K;

- an algorithm that reconstructs the object from its image;

- an algorithm that encodes the non-numerical class $\mathrm{K}$ as a whole.

Numerous publications on algorithmic theory of measurements are devoted to these problems. Mainly, they deal with consistent classifications and establishment of scales. There is a certain process which is determined by specific laws of the development of deductive theories and which is still to be investigated by metrologists. On the other hand, it was found that any sufficiently powerful theory cannot be resolved. Here are the natural limits of the axiomatic method.

\section{MEASUREMENT THEORY AS AN EDUCATIONAL FACTOR}

The measurement theory concentrates the knowledge about the status of measurement technology and instrumentation, reduces the immense quantity of metrological phenomena to a limited number of fundamental methods, principles and means and makes for the understanding of measurements. Solving measurement problems in a systematic and planned manner with the help of the measurement theory one can optimize their solutions, limit possible incorrect actions and spending and predict future requirements (Figure 5) [18].

Until now the educational programs and those of refresher courses were hardware-oriented. However, automation of measurements and modern technology can be introduced only if specialists will also be software-oriented. One must understand not only instruments but processes as well.

The software-oriented thinking is not restricted by the writing of computational programs and includes the following problems:

- choice of appropriate concepts and definitions;

- target-oriented exact formulation of measurement problems;

- discrimination between hardware-oriented and softwareoriented functions;

- discrimination between central and decentral functions;

- clearing-up of the relationships between the system operator and the measurement system, between measurement systems as well as between operators;

- automation of measurement processes after consideration of economic criteria, of the costs associated with measurements that were or were not carried out, with the measuring instrumentation that is 


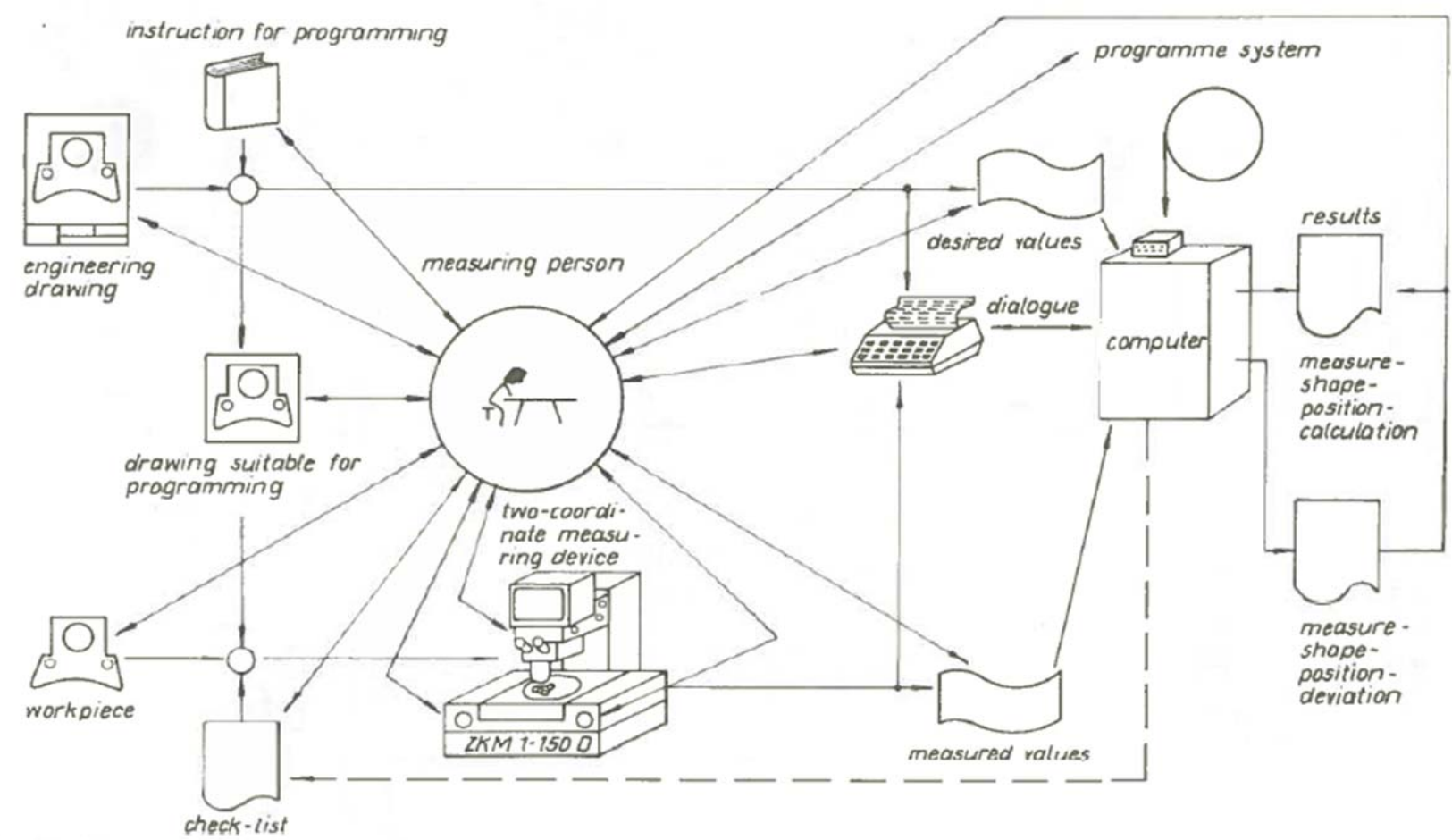

Figure 5. Working steps for computer-supported coordinate measurement, after [18].

already available or is to be purchased and with the training of the personnel.

The more productive is the measurement theory, the easier are solutions and the smaller is the educational expenditure. However, the ever-increasing training costs should not be underestimated. It is through education and training that the results of scientific research add to the efficiency of production (Figure 6).

\section{PROBLEM OF IMPROVING SYSTEMS OF UNITS}

The theory of building up a system of units, which could adequately meet complicated requirements of science and practice has become one of those few branches of general metrology, that was successfully developed and logically completed in 1954-1960 by accepting the unquestionably progressive International system of units (SI). However, some old unresolved problems have still remained in this field and new ones that require immediate solution have appeared since.

It can be explained by the fact, that the number of independent and derived measurands is constantly increasing. Now the international system of units already consists of 135 units of physical quantities and the number of parameters, measured in science and technology, can reach 700 in 1980 and increase up to 2000 in the nearest future, according to the existing forecast. Such a quantitative growth causes difficulties from the point of view of SI, whose aim is to establish simple relations between existing units, and suggests the necessity of introducing some alterations.

Thus, at present we are faced with the problem of reviewing the definition of the metre as the old definition doesn't allow to improve the accuracy of realization of the unit.

Till nowadays the definition of the unit of temperature (using the thermodynamic scale) is in a certain contradiction with its realization (using IPTS).

The problem of reviewing the definitions of most important units in the field of electricity and magnetism is an urgent one.
A number of fundamental questions of the theory of systems of units remains unsolved as well. So during several years a discussion about the status of angular units is underway. Some metrologists consider it necessary to speak out in favour of introducing the unit of plane angle into the number of basic units and placing the solid angle unit among derived units.

All these questions demand additional theoretical study and broad discussion by the scientific community.

\section{PROBLEM OF IMPROVING BASIC STANDARDS AND ESTABLISHING A SYSTEM OF INTERRELATED STANDARDS}

The problem of improving standards is closely related to the problem of improving the systems of units, though this relation is not a straightforward one. At present the change from standards, forming a certain set to a system of interrelated standards, based on stable physical phenomena and fundamental physical constants should be considered the main trend in this field. Undoubtedly first of all we must think about improving the standards of basic units. But it is not obligatory that a system of basic standards should coincide with the set of basic units. The system of standards of electrical units, where the main standards are the standard of the e.m.f. unit - the volt (and not the ampere) - and the standard of the farad can serve as an example of such a non-antagonistic difference. We are going to show below that such a structure of the system of standards is not in contradiction with the definition of the ampere as a basic unit of SI.

From the point of view of accuracy the best system would be such a system of standards, in which all the basic standards were "countable". At present the standard of the second (based on the counting of the number of periods of ${ }^{137} \mathrm{Cs}$ radiation) and the metre (based on the counting of the number of wavelengths corresponding to the orange line in ${ }^{86} \mathrm{Kr}$ radiation) are designed in such a way. The prospect of a changeover to the definition of the mass unit, according to which the kilogram 

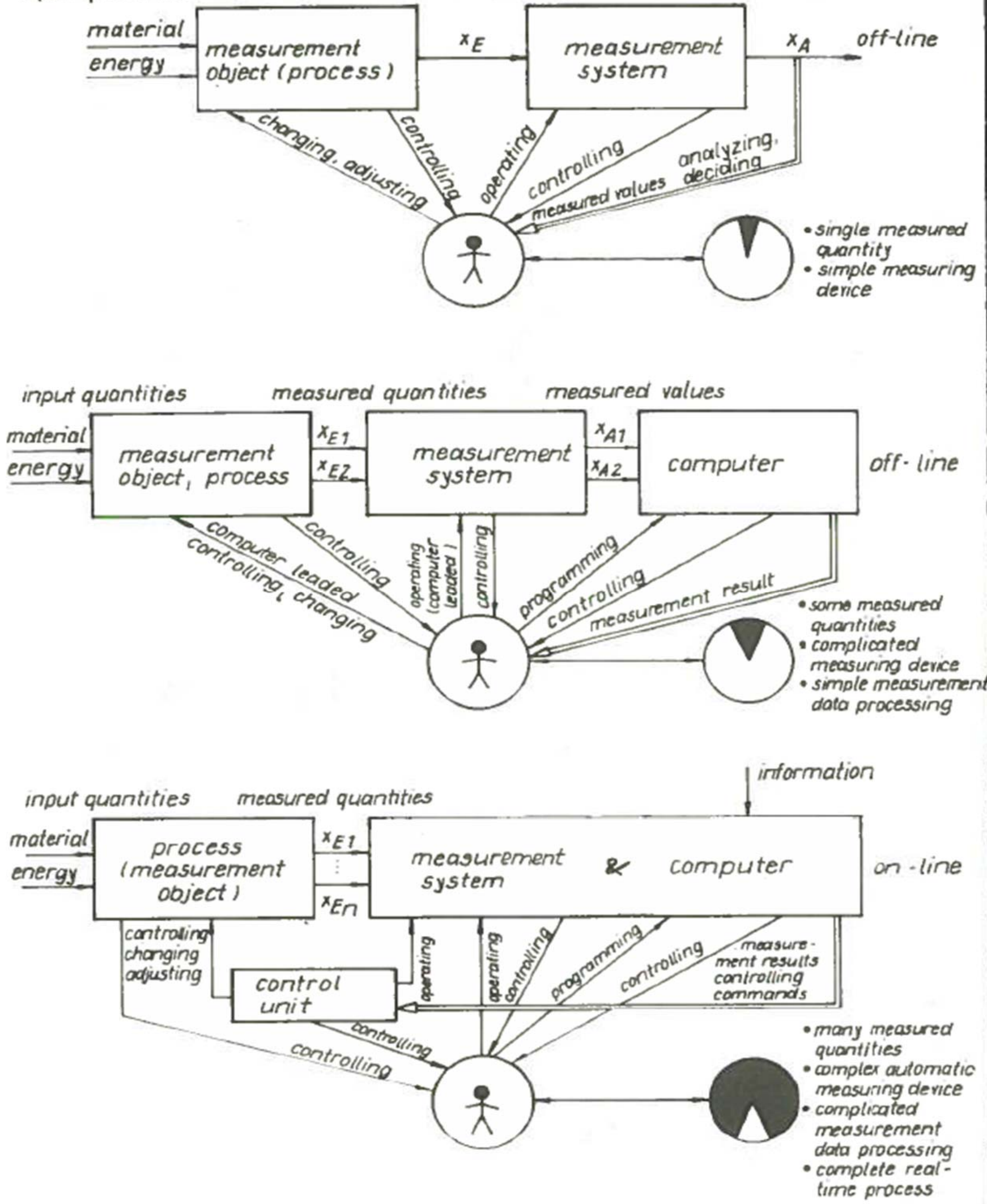

Figure 6. Development of measurement devices, working fields and educational requirements.

will represent the mass of countable number of atoms of a certain element, most probably the ${ }^{28} \mathrm{Si}$ isotope. The fact that there will be no less of accuracy in transferring the value of the unit into the field of molecular and atomic masses (though the accuracy of realization will be comparable with the accuracy of the existing standard or even lower) can be considered one of the main advantages of such a definition of kilogram. 
In principle it's also possible to design the standard of one of the electrical units on the basis of counting the number of elementary charges. However in spite of the fact, that modern technology allows to realize such counting the realization of a standard on such a basis is a matter of the future.

After the establishment of basic standards of a system on the basis of counting the set of values of fundamental physical constants (both dimensional and dimensionless) can be measured. The obtained values of constants should be mathematically processed in order to determine most reliable adjusted values and then the standards of derived units should be established on their basis. The system of such standards will be self-consistent and will not contradict the system of units.

The principle of building up such a system of standards as illustrated by the realization of the e.m.f. unit - the volt using the Josephson effect and the fundamental physical constants is given in [19] and besides will be considered in detail in a special report to the section "Metrological assurance". The aim of the creation of such a system is not only to establish a more objective internal interrelation and self-consistency of the standards of units of physical quantities, but also to improve the accuracy of all standards of the system.

The necessity of radical rearrangement of the system of standards (primary and secondary ones) for another basic unit the kelvin - through the transfer to the thermodynamic temperature scale from the International practical temperature scale is quite evident. It is due to the fact, the absolute temperature values are needed for fundamental physical research, as absolute thermodynamic temperatures come into physical equations. The problems associated with this go beyond the bounds of metrology per se. Thus, a further improvement of the gas thermometer aimed at practical realization of the thermodynamic temperature scale demands the development of the theory and methods of molecular dynamics, the theory of the virial equation of the state of real gases and more accurate determination of appropriate virial coefficients for pure gases and of the universal gas constant.

The improvement of the accuracy of the determination of the gas constant and the Avogadro number in its turn allows to have a more precise value for the Boltzmann constant and the second constant in the Planck radiation law, which is essential for building a high temperature thermodynamic scale and for many thermodynamic calculations.

On the other hand, the improvement of these constants allows to go over to a new method of realization of another basic unit - the candela - and to improve the whole system of standards in the field of photometry in a wide spectral range using the "black body" radiation and to effect the change-over to the energy photometry.

\section{PROBLEM OF DEVELOPING “NATURAL" STANDARDS AND USING FUNDAMENTAL PHYSICAL PHENOMENA}

This problem, that arose at the moment when it was felt that the international uniformity of measurements, should be ensured, reflects the natural and therefore constant tendency among metrologists to use the most stable natural phenomena for the realization of units of physical quantities, that is to establish reproducible standards.

100 years of intensive investigations of metrologists in this direction have resulted in the establishment of natural standards of the above mentioned length unit and time unit. In recent years the standards of magnetic induction (for high fields), angular velocity, etc. whose operating principles are based on relatively easily reproducible natural phenomena. At present "natural" standards are being developed in the following fields: of thermometry (the use of the phenomenon of the nuclear quadrupole resonance), of voltage measurements (the effects of Josephson, Stark and Pockels), of radiation energy (the monochromaticity of atomic and nuclear transitions), etc.

The following two common trends can be rather clearly seen in all these studies:

- a wide use of quantum effects which even resulted in the coining of the term "quantum metrology";

- the use of fundamental physical constants that are most stable realizations of natural laws as references in standards.

In this connection, the problem of a more accurate determination and adjustment of fundamental physical constants is in itself becoming very important. It was shown above what is the role of this problem in the establishment of an interrelated system of standards. If one also takes into account the fact that the accuracy of physical constants characterizes the depth of our notions of physical phenomena and laws interrelating them, then it can be said with confidence that everybody - from physicists and metrologists to practical engineers - is interested in the improvement of accuracy of fundamental physical constants. In this respect, the work done at VNIIM has shown that, from the point of view of the progress of metrology, in the first place it is necessary to improve the accuracy of the constants involved in the realization of the volt using the Josephson effect.

As to the other trend in the improvement of standards, i.e. the trend of a wide use of quantum effects and phenomena that are not substantially influenced by environmental factors, the use of the superconductivity phenomenon is the most characteristic feature of the present stage.

This phenomenon has attracted a special attention of metrologists after it was shown that superconductivity presents a unique manifestation of the existence on a macroscopic scale of such quantum mechanical phenomena as the Meissner effect (of ideal diamagnetism), the phenomenon of quantizing the magnetic flux in superconductors with multiple links, a whole class of contact phenomena united by the common name "Josephson effects" as well as the phenomenon of quantum interference of currents in superconductors which is related to the latter effects.

The ideal diamagnetism of superconductors provides a unique opportunity for the development of magnetic suspensions and bearings that will undoubtedly enhance the improvement of high-precision measuring instrumentation in the field of mechanical measurements where the total absence of friction is of exclusive importance.

The main (classical) property of superconductors (the absence of dissipative losses, i.e. the ideal conductivity) together with the Meissner effect (ideal diamagnetism) have long ago led to the idea of constructing superconducting magnets with high values of induction and highly uniform magnetic fields that practically do not consume any energy. At present $7.5 \mathrm{~T}$ magnets have been produced with a field inhomogeneity of less than $2 \times 10^{-7}$ in a $0.5 \mathrm{~cm}^{3}$ volume (England). Such a magnet made a resolution of approximately $5 \times 10^{-9}$ possible. The work on the development of new materials having high superconducting transition temperatures and stable structures that is underway in the whole world allows to expect the emergence of magnets with field inductions up to 20 to $30 \mathrm{~T}$.

Various quantum-mechanical properties of superconductors 
(from ideal diamagnetism to the Josephson effects) open the prospects (and are already used) for the development of superconducting gravimeters for relative measurements of gravity that have an extremely high sensitivity. The theoretical limit of one of the designs of such gravimeters proposed by NBS [20] is $\sim 10^{-14} \mathrm{~g}$.

A dilatometer based on the principle of superconducting cavity resonator and having a sensitivity of $\sim 10^{-14} \mathrm{~m}$ was built at the Institute of Physical Problems of the USSR Academy of Sciences. The gravimeter which is an embodiment of this principle makes it possible to obtain a sensitivity of $\sim 10^{-10} \mathrm{~g}$ for averaging time of $\sim 1$ minute.

Apparently, widest prospects for the use of unique properties of superconductors are open in the fields of electrical and magnetic measurements. Already at present Josephson volt standards are used in the majority of metrological laboratories of the world for the maintenance of the volt value with an accuracy $\sim(3-5) \times 10^{-8}$. This brings about, in particular, an important task of developing a transportable transfer standard for the e.m.f. unit in which all achievements of physics and low-temperature technology should be used.

Numerous applications will be found for superconducting quantum magnetometers using the phenomenon of quantum interference of currents and Josephson effects. The sensitivity of the transducers of these magnetometers (SQUIDs) may be close to the fundamental limit whose value can be estimated as

$$
\frac{\delta \Phi_{\mathrm{x}}}{(\Delta \mathrm{F})^{1 / 2} \min } \approx 10^{-8} \frac{\Phi_{0}}{\mathrm{~Hz}^{1 / 2}}
$$

where

$\delta \Phi_{\mathrm{X}}$ is the minimum detected change in the magnetic flux;

$\Delta \mathrm{F}$ is the frequency bandwidth of the signal;

$\Phi_{0}$ is the magnetic flux quantum equal to $2 \times 10^{-15} \mathrm{~Wb}$.

These magnetometers are beyond competition in respect of their sensitivity and accuracy as compared with all other types of magnetometers in the field of measurement and stabilization of low and medium magnetic fields. Of no less importance is the fact that together with other superconducting devices, such as current comparators, magnetic flux transformers, super conducting screens, these magnetometers provide great opportunities for increasing the sensitivity and accuracy of a wide class of electrical measuring devices. In particular, they make feasible measurements of voltages of the order of $10^{-12}$ to $10^{-15} \mathrm{~V}$ comparisons of d.c. currents with a relative uncertainty of $10^{-8}$ to $10^{-10}$, inductance comparisons with a sensitivity of the order of $10^{-14} \mathrm{H}$, attenuation measurements at radio frequencies with a relative uncertainty of $10^{-3}$ and in a number of other measurement fields requiring high accuracy. We should also note that among the promising fields are applications of superconducting cavities for frequency stabilization of microwave oscillators (at present relative frequency instabilities of $1.2 \times 10^{-12}$ for one hour and $1.2 \times 10^{-13}$ for 10 seconds have been achieved) and the use of non-linear properties of Josephson junctions for multiplication of frequencies in the $3-\mathrm{cm}$ band up to the far infrared part of the spectrum which is of great importance for bridging a gap between the frequencies of cesium clocks and lasers.

\section{CONCLUSIONS}

Even this brief review of individual problems, that are most important for the development of metrology and measurement technology and instrumentation, shows that metrology (including the general theory of measurements) is a rapidly developing field of scientific knowledge, i.e. a science whose progress will undoubtedly enhance further progress in various branches of industry and scientific research and whose actual formation can be promoted by concerted efforts of metrologists in collaboration with scientists and practical workers in various fields.

\section{REFERENCES}

[1] V.D. Komarov, Nauchno-tehnicheskaja revoljuzia I nekotorye, metodologicheskye problemy tehnicheskih nauk (scientifictechnical revolution and some methodological problems of technical sciences). Leningrad 1970.

[2] D. Hofmann, Handbuch Messtechnik und Qualitätssicherung (Handbook Measurement Engineering and Quality Assurance) Berlin: VEB Verlag Technik 1979.

[3] W. Gutjahr, Die Messung psychischer Eigenschaften (Measurement of psychological properties). Berlin: VEB Deutscher Verlag der Wissenschaften 1971.

[4] P.S. Sawelski, Die Masse und ihre Messung (Mass and its measurement). Moscow: MIR, Leipzig: VEB Fachbuchverlag 1977.

[5] G. Hofmann, Effektivitätssteigerung durch Monosemierung der Fachsprache der Messtechnik (Improving Efficiency by using monosemantic term in the terminology of measurement engineering). Feingertetechnik 28(1979) H. 5, S. 216-218.

[6] M. Peschel, Modellbildung für Signale und Systeme (Models for signals and systems). Berlin: VEB Verlag Technik 1978.

[7] N.A. Krinickij, Algoritmy vokrug nas (Algorithms around us). Moscow: Nauka 1977.

[8] D. Hofmann, Heuristische Algorithmen zur Rationalisierung von Messprozessen (Heuristic algorithms for the rationalization of measurement processes). messen - steuern - regeln 14(1971) H.5, S. 172-174.

[9] F.H. Lange, Methoden der Messstochastik (Methods of stochastical measurements). Berlin: Akademie Verlag 1978.

[10] G.I. Kavalerov, Mandelstam, S.M.: Vvedenie v informacionnuyu teoriyu izmerenii (Introduction in measurement-information theory). Moscow: Energija 1974.

[11] L. Finkelstein, Instrument science. Introductory article. Journal of Physics E: Scientific Instruments 10 (1977) S. 566-572.

[12] D. Bosman, Instrument science. Systematic design of instrumentation systems. Journal of Physics E: Scientific Instruments 11(1978) S. 97-105.

[13] A.P. Stachov, Vvedenie v algoritmicheskuyu teoryu izmerenija (Introduction in algorithmic measurement theory). Moscow: Sovetskoye radio 1977.

[14] P.R. Halmos, Measure Theory. New York, Heidelberg, Berlin: Springer Verlag 1974.

[15] J. Pfanzagl, V. Baumann, H. Huber, Theory of Measurement, 2nd. ed. Würzburg, Wien: Physica Verlag 1971.

[16] L. Finkeistein, The relation between the formal theory of measurement and pattern recognition, in: Practical Measurement for Improving Efficiency. ACTA IMEKO 1976. Budapest: Akademiai Kiado 1977.

[17] Y. Morita, A. Kobayashi, H. Iida, M. Ishigami, Of classification and industrial measurement, in: Practical Measurement for Improving Efficiency. IMEKO VII. Preprint Vol. 3, 1976.

[18] W. Lotze, M.W. Hartmann, Maus (2D) Programmsystem zur Berechnung von Mass, Form und Lage punktweise gemessener ebener Werkstücke. Nutzerbeschreibung (Maus (2 D) Programmsystem for the calculation of measure, shape and position pointlike measured plane objects). Dresden: Technical University 1977.

[19] Y.V. Tarbeev, Izvestija AN SSSR. Ser. Energetika i transport, 1978, N. 3.

[20] R.P. Giffard, J.C. Gallop, B.W. Petley, Progress in quantum electronics, 1978, vol. 4, p.4. 